\title{
Understanding and modeling space-time Holocene climate variability
}

\author{
Thomas Laepple', R.V. Donner ${ }^{2}$ and T. Kunz' \\ Potsdam, Germany, 25-27 October 2017
}

PAGES' Climate Variability Across Scales (CVAS) working group aims to develop a thorough understanding of the mechanisms underlying climate variability across temporal and spatial scales. A specific focus lies on centennial to millennial timescales, where the variability observed in paleoclimate archives cannot be exclusively explained by a linear response to external factors, like solar variability or changes in orbital parameters, but exhibits a rich variability across a broad range of scales. In this context, the first CVAS workshop in fall 2016 (Franzke 2017) identified an existing knowledge gap regarding the scaling regimes of climate variability during the Holocene and the associated differences between tropics, extratropics and polar regions, as well as between land and ocean.

The purpose of the second CVAS workshop, held at the Potsdam Institute for Climate Impact Research, was to specifically address these aspects to characterize Holocene climate variability in both space and time (Fig. 1). With relatively stable climate conditions similar to present-day, the Holocene constitutes a unique reference period for studying temporal scaling properties based upon a variety of paleoclimate archives, together with reconstructions of key climate forcing factors like solar irradiation and volcanic activity.

With generous financial support from PAGES, the Alfred Wegener Institute Helmholtz Center for Polar and Marine Research, and the German Federal Ministry for Education and Research, the meeting brought together 41 scientists, including 25 early-career researchers, working on Holocene climate variability from three distinct perspectives: data acquisition and interpretation, development and application of time-series analysis methods, and theoretical climatology and climate modeling.

The first day comprised plenary talks introducing the state of the art along with key open challenges in the field. These overview presentations and accompanying panel discussions set the stage for forming three breakout groups:

\section{Data - how to select the best archives to quantify climate variability and understand their caveats}

The data group summarized key properties of the major climate archives concerning po tential time-scale dependencies in the proxyto-climate relationship and the time-scale

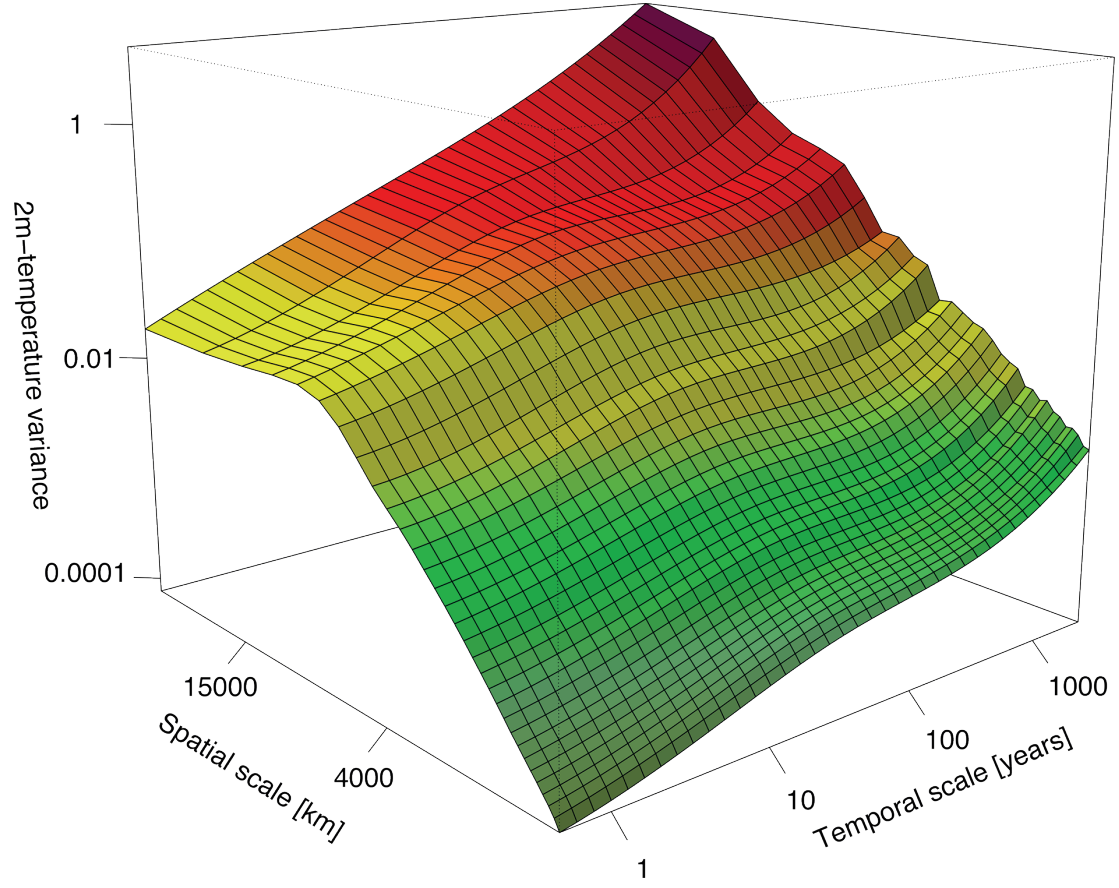

Figure 1: Space-time spectrum of simulated Holocene temperature variability. Global $2 \mathrm{~m}$ temperature anomaly fields from the last 7000 years of the TraCE-21 ka paleoclimate model simulation (Liu et al. 2009) have been decomposed into spherical harmonics; the annual cycle has been removed. The power spectral density generally increases towards longer spatial and temporal scales. dependencies of non-climatic effects on the recorded signal. It further identified key areas and datasets that provide good starting points to characterize the spatial and temporal scaling of climate variability.

\section{Analysis tools - from paleo-} observations to robust and reproducible variability estimates

The subgroup on analysis tools identified a suite of traditional as well as unconventional methods for studying temporal and spatial scaling in paleoclimate archives and discussed conceptual and practical limits of the existing methods. Particular attention was paid to the challenge of unevenly sampled and "gappy" data and possible ways to overcome existing methodological limitations, triggering some joint research efforts among different members of this subgroup.

\section{Models and theory - origins and interpretation of the space-time structure of Holocene climate variability}

The model and theory subgroup discussed possible mechanisms that may generate centennial to millennial scale variability and shape its temporal and spatial structure summarized existing theoretical concepts to describe and understand such fluctuations, and then formulated working hypotheses in order to focus ongoing research efforts. The subgroup also emphasized the necessity to communicate to the data subgroup the specific data requirements for testing and selecting different theoretical hypotheses.

Following the discussions within the subgroups and in the panel, initiatives have been started to systematically summarize the current state of the art in upcoming review papers. Another outcome of these discussions was a dedicated CVAS session and short course at the General Assembly of the European Geosciences Union in Vienna, Austria, in April 2018.

\section{AFFILIATIONS}

'Alfred Wegener Institute Helmholtz Centre for Polar and Marine Research, Potsdam, Germany

2Potsdam Institute for Climate Impact Research, Potsdam, Germany

\section{CONTACT}

Thomas Laepple: tlaepple@awi.de REFERENCES

Franzke C (2017) PAGES mag 25: 128 Liu Z et al. (2009) Science 325: 310-314 